\title{
Direct identification of Monilinia brown rot fungi on infected fruits by matrix-assisted laser desorption/ionization (MALDI) mass spectrometry
}

\author{
Florian Matthias Freimoser ${ }^{1 *} \mathbb{B}$, Maja Hilber-Bodmer ${ }^{1}$, René Brunisholz ${ }^{2}$ and David Drissner ${ }^{3}$
}

\begin{abstract}
Background: Brown rot of stone and pome fruit is a serious fungal disease that is mainly caused by four species in the genus Monilinia. Of these four species, Monilinia fructicola is the most devastating pathogen and of particular concern because it undergoes sexual recombination and has recently been introduced to Europe. So far, Monilinia diagnosis required a multiplex PCR analysis and gel electrophoresis. In contrast, intact-protein biotyping by mass spectrometry is considerably faster and cheaper. However, it usually requires an in vitro cultivation step prior to the MALDI analysis. It was thus attempted to establish a method for the identification of Monilinia species by MALDI biotyping with fungal material derived directly from infected fruits; without an in vitro cultivation step.

Results: To simplify and render MALDI biotyping of fungi more reliable, an improved protocol for the preparation of crude protein extracts and for collecting MALDI-TOF MS data for biotyping was developed. We generated reference spectra for all four Monilinia brown rot fungi and were able to reliably identify Monilinia species based on fungal material that was collected directly from infected fruits. This method allowed the correct, fast and economic identification of M. fructicola and M. laxa, while M. fructigena and M. polystroma could not be distinguished reliably.
\end{abstract}

Conclusions: MALDI biotyping may be used as an economical tool for the routine diagnosis of Monilinia brown rot fungi on infected fruits.

Keywords: Biotyping, Intact-protein profiling, MALDI-TOF/TOF MS, Monilinia

\section{Background}

Brown rot of stone and pome fruit is a fungal disease that causes economic losses and limits fruit production worldwide [1]. The causative agents of this disease are mainly four species in the genus Monilinia. Although the four Monilinia brown rot fungi exhibit host preference, all are able to infect and cause severe damage in various members of the Rosaceae family. The most common of these pathogens worldwide is Monilinia laxa (Aderh. \& Ruhland) Honey 1945 (Ascomycetes, Helotiales) [2], which also causes twig and branch blight and

\footnotetext{
*Correspondence: florian.freimoser@agroscope.admin.ch

${ }^{1}$ Agroscope, Institute for Plant Production Sciences IPS,

Schloss 1, 8820 Wädenswil, Switzerland

Full list of author information is available at the end of the article
}

is one of the most important postharvest pathogens of stone fruits [3]. Monilinia fructigena Honey ex Whetzel 1945 (Ascomycetes, Helotiales) occurs worldwide and causes damage in pome fruit [3, 4]. Monilinia polystroma van Leeuwen [5] (Ascomycetes, Helotiales), distinguished by molecular and morphological means from $M$. fructigena $[5,6]$, is mainly found in Asia and Europe [5, 7]. Brown rot caused by M. fructicola (G. Winter) Honey 1928 (Ascomycetes, Helotiales) is one of the most devastating stone fruit diseases in Asia, Australasia, North and South America [3, 8]. This species regularly undergoes sexual recombination, which may promote the development and establishment of fungicide-resistant strains [9-11]. In Europe, this disease was described for the first time in 2001, in southern France, 
and since then the pathogen has spread [12-15]. The European Plant Protection Organization recommends M. fructicola for regulation as a quarantine pest [16], even though it is widespread now. Local survey and management schemes are thus advised, because phytosanitary measures and chemical control can prevent the establishment of this pathogen [14]. To expedite the identification of $M$. fructicola, we strove for a fast and reliable matrix-assisted laser desorption/ionization time-of-flight mass spectrometry (MALDI-TOF MS) method that does not require prior in vitro cultivation of the pathogen.

Monilinia brown rot fungi exhibit slight morphological variation, but their identification and delineation usually includes a polymerase chain reaction (PCR)-based analysis [17]. Although this analysis can be performed with fungal material obtained directly from infected fruits (i.e. does not require a cultivation step), it involves DNA extraction, a lengthy PCR protocol and performing gel electrophoresis. Monilinia polystroma differs only by five base transitions in the ITS region from $M$. fructigena [6] and thus requires multiplex PCR and the resolution of fragment size differences of $23 \mathrm{bp}$ [7]. In contrast to the DNA-based identification, microbial biotyping builds upon the observation that MALDI-TOF MS of entire microbial cells or of crude extracts reveals species-specific profiles of molecular masses [18]. Most of the mass peaks detected by this method, also called MALDI biotyping, are attributed to basic, abundant and conserved proteins, particularly ribosomal proteins $[19,20]$ and to a certain degree to cell wall-associated proteins [21]. Sample preparation for MALDI biotyping is considerably faster and cheaper than for the DNA-based methods and the measurement itself is performed within a few minutes. However, MALDI biotyping requires pre-existing reference spectra for the species to be identified. For clinically relevant microorganisms, comprehensive databases of reference spectra exist, but organisms from agricultural ecosystems are often not included in commercially available databases. Furthermore, the method performs reliably for many bacteria, while the MALDI-TOF MSbased identification of fungi is often more difficult and requires optimization of extraction and data acquisition protocols. Finally, a major limitation for the rapid identification of bacteria and fungi is the in vitro cultivation step, which is usually required prior to the MALDI-TOF analysis.

Recently, the potential of different electrophoresis methods and of MS for the identification of Monilinia species based on proteins was assessed [22]. The authors successfully used MALDI-TOF MS to identify the four closely related Monilinia brown rot species when they were grown in vitro. However, identification of fungal material obtained directly from infected fruits was not possible, because the host affected the result of the MALDI-TOF MS analysis and prevented a reliable identification at the species level. To circumvent this unreliability, a combination of MALDI-TOF MS analysis and capillary isoelectric focusing (CIEF) or capillary zone electrophoresis (CZE) was suggested [22]. It was the goal of the project presented here to build upon this work and to establish the identification of Monilinia species by MALDI biotyping with fungal material derived directly from infected fruits; without an in vitro cultivation step. We prepared crude, acid protein extracts from minute sample amounts with glass beads, generated reference spectra for several isolates of all four Monilinia brown rot fungi, and were thus able to identify Monilinia species with material collected directly from infected fruits. This method allowed the correct, fast and economic identification of M. fructicola and M. laxa, while M. fructigena and $M$. polystroma could not be distinguished reliably. MALDI biotyping may thus be used as an economical tool for the routine diagnostic of Monilinia brown rot fungi on infected fruits.

\section{Methods}

\section{Fungal isolates and cultivation}

All Monilinia isolates were obtained from infected apricots or peaches as described previously [11] and grown on Difco ${ }^{\mathrm{TM}}$ potato dextrose agar (PDA; Becton, Dickinson and Company, Le Pont de Claix, France) at $20{ }^{\circ} \mathrm{C}$ in the dark. The Monilinia isolates used for this study are listed in Table 1.

\section{Fruit infection experiments}

Ripe, harvested cherries, prunes or apples were wiped with $70 \%$ ethanol and dried. Monilinia mycelium and conidia were harvested with a sterile toothpick from an agar plate (1-2 weeks old). To start the infection process, healthy fruits were pierced with the toothpick. Fruits infected in this manner were either stored in disposable, transparent plastic containers (cherries and prunes) or in transparent plastic bags (apples), kept at $20{ }^{\circ} \mathrm{C}$, and inspected daily. Fruits with clear signs of infection and developing mycelium were removed and the fungus causing the infection was identified by DNA-based methods or by MALDI biotyping as described below.

\section{DNA-based identification}

Mycelium was harvested with a wooden toothpick and DNA was extracted with $50 \mu \mathrm{l}$ Kawasaki buffer as described [11, 23]. The four Monilinia species were identified by a multiplex PCR assay as described by Coté et al. [17]. The PCR conditions were the following: initial denaturation at $95{ }^{\circ} \mathrm{C}$ for $15 \mathrm{~min}, 28$ cycles of $94{ }^{\circ} \mathrm{C}$ for 
Table 1 Monilinia isolates used for generating the reference main spectra (MSP) for this study

\begin{tabular}{lllll}
\hline Species & Isolate & Origin & Year & Host \\
\hline M. fructicola & 11SD14 & Switzerland & 2011 & Apricot \\
& M16 & USA & 2003 & Peach \\
& AS5 & France & 2003 & Apricot \\
& IT3 & Italy & 2008 & Peach \\
M. laxa & SD14 & Switzerland & 2010 & Apricot \\
& E134.5 & Switzerland & 2009 & Apricot \\
& 10S1 & Switzerland & 2010 & Apricot \\
& $10 M 14$ & Switzerland & 2010 & Apricot \\
& E139.5 & Switzerland & 2009 & Apricot \\
M. fructigena & E57.7 & Switzerland & 2009 & Apricot \\
& 113. A.15.2 & Switzerland & 2010 & Apricot \\
& 180 & Switzerland & 2008 & Apricot \\
& E71.1 & Switzerland & 2009 & Apricot \\
& 113 A8 & Switzerland & 2010 & Apricot \\
M. polystroma & Q1 & Switzerland & 2009 & Apricot \\
& P16 & Switzerland & 2009 & Apricot \\
& C6 & Switzerland & 2009 & Apricot \\
& G4 & Switzerland & 2010 & Apricot \\
& & &
\end{tabular}

Strains were isolated from infected apricots or peach in the years 2003 or 20082011 and maintained on PDA medium. The strains were previously identified and characterized molecularly (Hilber-Bodmer et al. [7], Jänsch et al. [11])

$1 \mathrm{~min}, 60{ }^{\circ} \mathrm{C}$ for $1 \mathrm{~min}, 72^{\circ} \mathrm{C}$ for $1 \mathrm{~min}$; final extension at $72{ }^{\circ} \mathrm{C}$ for $10 \mathrm{~min}$. Alternatively, the fungal ITS region was amplified with primers ITS1f [24] and ITS4 [25] and PCR products were directly used for sequencing.

\section{Protein extraction for MALDI-TOF MS-based identification}

For protein extraction, a small amount of mycelium (approx. 1-5 × 1-5 mm), either from a PDA plate or from an infected fruit, was collected with a toothpick and transferred to $1 \mathrm{ml} 70 \%$ ethanol. After vigorous shaking, the fungal material was pelleted, the supernatant discarded and the mycelium dried in a concentrator (room temperature, 60 min; Eppendorf, Hamburg, Germany). The pellet was resuspended in $25 \mu \mathrm{l}$ formic acid (70\%, Carl Roth $\mathrm{GmbH}$, Karlsruhe, Germany), glass beads (0.5$0.75 \mathrm{~mm}$, approx. $50 \mu \mathrm{l}$; Retsch $\mathrm{GmbH}$, Haan, Germany) were added and samples were shaken (Eppendorf Thermomixer, $1400 \mathrm{rpm}$, room temperature, 15 min; Eppendorf). Following the addition of $25 \mu$ l acetonitrile (Carl Roth $\mathrm{GmbH}$ ), samples were mixed, centrifuged and $1 \mu \mathrm{l}$ of the supernatant was applied to MALDI-TOF steel target plates (Bruker Daltonics GmbH, Bremen, Germany). After air drying, the spots were overlaid with $1 \mu \mathrm{l}$ matrix ( $\alpha$-cyano-4-hydroxycinnamic acid, HCCA, $10 \mathrm{mg} /$ $\mathrm{ml}$ in acetonitrile-water-trifluoroacetic acid (TFA) (50:47.5:2.5); HCCA from Sigma-Aldrich Chemie $\mathrm{GmbH}$, Steinheim, Germany, or Bruker Daltonics GmbH; TFA from Merck KGaA, Darmstadt, Germany). Alternatively, the fungal extract was stored at $-20{ }^{\circ} \mathrm{C}$ for later analysis.

\section{MALDI-TOF MS measurement}

MALDI-TOF MS spectra were acquired with a microflex LT MALDI-TOF mass spectrometer (Bruker Daltonics $\mathrm{GmbH}$ ). Data collection parameters were defined using flexControl software (Version 3.4; Bruker Daltonics $\mathrm{GmbH}$ ). Laser frequency of $60 \mathrm{~Hz}$ in the positive linear mode with mass acquisition ranging from 2 to $20 \mathrm{kDa}$. Final spectra consisted of 500 shots per spot (random walk, 50 shots per raster spot). No peak exclusion criterion was defined, but the resolution was required to be higher than 100 and the measurement was quit if 20 subsequently failed judgements were encountered. Data collection was terminated as soon as 10 peaks with an intensity of $\geq 2000$ were detected. The laser intensity was adjusted to obtain spectra with maximal absolute peak intensities ranging from about $5 \times 10^{3}-10^{4}$ arbitrary units. Extracts that did not reveal any peak with these settings were discarded. The spectra were evaluated using the accompanying MALDI BioTyper OC software (Version 3.1) with standard settings, but without intensity correction (Bruker Daltonics GmbH). According to the BioTyper software, a score of $\geq 2.0$ represents an identification at the species level and values between $\geq 1.7$ and $<2$ at the genus level. Measurements with a score $<1.7$ were counted as "not identified".

\section{Creation of MALDI-TOF MS reference spectra}

To generate MALDI-TOF main spectra (MSP), eight replicates of a fungal protein extract were spotted and each spot was measured three times. The 24 MALDI-TOF MS spectra were visually inspected and the 20 best spectra were used to calculate an MSP with the default parameter set of the BioTyper MSP Creation Standard Method (Max. mass error of each single spectrum 2000; desired mass error for the MSP 200; desired peak frequency $25 \%$; max. desired peak number for the MSP 70). The MSP of the 18 Monilinia isolates used for this study (see Table 1) were clustered based on Pearson correlation coefficients and UPGMA network creation (BioNumerics 7.5, Applied Maths, Sint-Martens-Latem, Belgium) (see also Fig. 1a).

\section{ZipTip preparation of protein extracts for subsequent MALDI-TOF/TOF MS analysis}

For the preparation of protein extracts prior to MALDITOF/TOF experiments, proteins were desalted and concentrated with C18-ZipTips (Millipore, Bedford, USA). Wetting solution contained $100 \%$ acetonitrile, equilibration solution and wash solution contained $0.1 \%$ TFA in water, and elution solution contained $0.1 \%$ TFA in acetonitrile-water (50:50). The original extract was spiked 


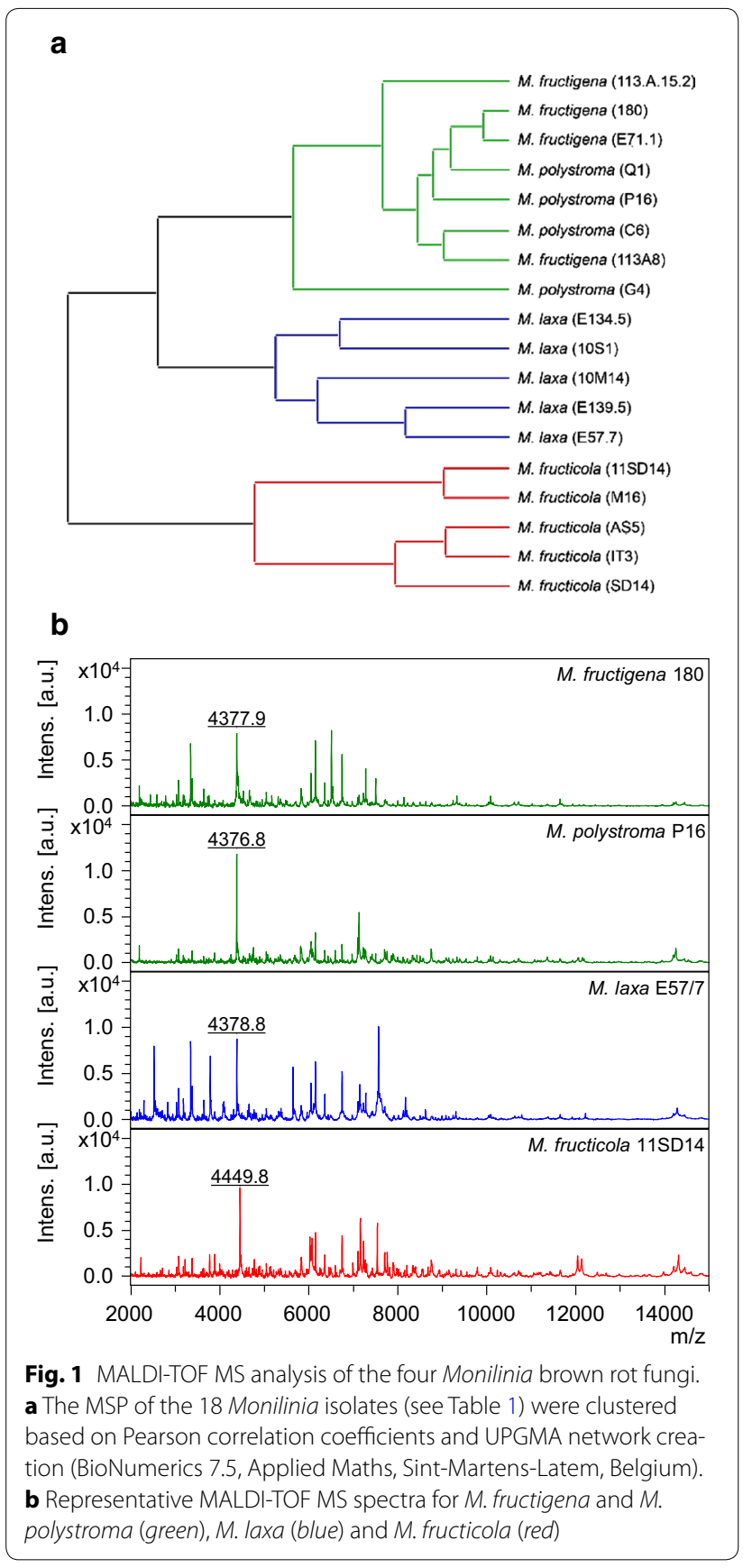

with $50 \mu \mathrm{l} 0.1 \%$ TFA in water, concentrated to a volume of $30 \mu$ l using a SpeedVac (Eppendorf) and subsequently applied to the ziptip sample preparation according to the manufacturer's protocol (Millipore, USA).

\section{MALDI-TOF/TOF MS for in-depth characterization of distinct mass peaks}

MALDI-TOF/TOF experiments were performed on an ultrafleXtreme mass spectrometer (Bruker Daltonics $\mathrm{GmbH})$. The instrument was equipped with a modified neodymium-doped yttrium aluminium garnet (Nd:YAG) laser (smartbeam II) operating at a wavelength of $355 \mathrm{~nm}$ and a frequency of $1 \mathrm{kHz}$. Mass spectra of intact proteins were recorded at matrix-optimized laser fluency in positive ion reflectron mode within a $\mathrm{m} / \mathrm{z}$ range of 400-5000, applying an acceleration voltage of $25 \mathrm{kV}$. Spectra were generated by accumulating data from approximately 10,000 laser shots. The mass spectra were externally calibrated using the peptide standard mixture II from Bruker Daltonics GmbH. Monoisotopic masses $m / z=4373.3$ and $m / z=4442.0$ were selected for fragmentation.

MALDI-TOF/TOF tandem mass spectrometry was performed using the LIFT technology [26] over a $\mathrm{m} / \mathrm{z}$ range of 20-4500 in the positive ion mode. The MALDI target plate was prepared by pipetting $0.7 \mu \mathrm{l}$ of eluted ziptip protein samples onto the target spots, which were overlaid with $0.7 \mu$ matrix [HCCA (Bruker Daltonics $\mathrm{GmbH}$ ), $2 \mathrm{mg} / \mathrm{ml}$ in acid (TFA) (50:49.9:0.1)]. All mass spectra were acquired and processed using flexControl (version 3.4, build 135) acetonitrile-water-trifluoroacetic and flexAnalysis (version 3.4, build 76), respectively (Bruker Daltonics $\mathrm{GmbH}$ ).

\section{Results \\ MALDI-TOF MS analysis of Monilinia brown rot fungi} cultures

In order to compare different Monilinia brown rot fungi by MALDI biotyping, crude formic acid/acetonitrile protein extracts of $M$. fructicola (5 isolates), M. fructigena (4 isolates), M. laxa (5 isolates) and M. polystroma (4 isolates) were prepared and used to generate reference main spectra (MSP). The 18 MSP were used to generate a MSP dendrogram, which revealed well-separated clusters for M. fructicola and M. laxa, and an unresolved branch containing the M. polystroma and M. fructigena spectra (Fig. 1a).

The individual MALDI-TOF MS spectra of the four Monilinia species revealed reproducible and distinct mass peaks (Fig. 1b). Most noteworthy was a strong signal with a molecular mass between 4370 and $4450 \mathrm{Da}$ that was particularly prominent in $M$. fructicola, $M$. fructigena and M. polystroma (Fig. 1B). Interestingly, this mass peak appeared at approx. $4450 \mathrm{Da}$ for M. fructicola, while for M. fructigena and M. polystroma the detected mass was approx. 80 Da lower. The MALDI-TOF MS spectrum for $M$. laxa, in contrast to the other three Monilinia species, featured several highly intense masses between 2 and $8 \mathrm{kDa}$. In this species, the peak at $4380 \mathrm{Da}$ was therefore less remarkable.

The newly generated MSP for four Monilinia brown rot fungi, based on material from in vitro cultures, thus clearly delineated three groups: $M$. fructicola, $M$. laxa and M. fructigena/M. polystroma. Since the latter two 
species were not well-separated, these two species were treated as an unresolvable complex in all following analyses. MALDI biotyping experiments where a $M$. fructigena isolate was identified as $M$. polystroma, or vice versa, were thus not counted as wrong identifications (Table 2).

The MSP reference database consisting of the MALDITOF MS spectra of 18 Monilinia isolates was then used to biotype 88 fresh extracts obtained from in vitro cultures of $M$. fructicola (30 extracts), $M$. laxa (22 extracts) and $M$. fructigena/M. polystroma (36 extracts) (Table 2). None of the extracts was identified as another genus than Monilinia. In total, 4 protein extractions failed (i.e. no identification), one identification was wrong and the remaining 83 identifications were correct (4 with a score between 1.7 and 2.0, 79 with a score $>2.0$ ). This analysis of closely related Monilinia brown rot fungi thus confirmed that MALDI-TOF MS is capable of discriminating M. fructicola, M. laxa and $M$. fructigena/M. polystroma in vitro cultures and may thus serve as a fast, reliable and economic alternative to the DNA-based identification of these plant pathogens.

\section{Identification of Monilinia species on infected fruits}

The MALDI-TOF MS-based identification of pathogens requires specific mass peak patterns and, preferably, protein extracts that are free from contaminations of host tissue. Consequently, in most cases an in vitro cultivation step is necessary in order to generate a pure culture from which appropriate extracts can be obtained. In order to facilitate and speed up the MALDI-TOF MS-based identification of Monilinia brown rot species, it was attempted to use fungal material obtained directly from infected fruit for preparing the protein extracts.
Ripe cherries, prunes and apples were infected with different Monilinia isolates (Table 2) and incubated until signs of colonization were apparent (1-2 weeks, depending on the isolate and fruit). All isolates were able to colonize the three types of fruit and formed mycelium on the fruit surface (Fig. 2a). The negative controls (uninfected fruits) never developed any sign of infection by Monilinia species. 130 extracts were prepared from infected apples, 47 extracts from infected prune and 56 extracts from infected cherries (Table 2). Overall, the MALDI-TOF MS spectra acquired directly from infected fruits resembled the spectra obtained from pure cultures of the four Monilinia brown rot fungi (Fig. 2b) and led to the correct identification (Table 2). Six and seven extractions of fungal material from apple or prune, respectively, failed (i.e. no identification), while all extractions of fungal material from cherry were successful. The only wrong identifications were observed for fungal extracts from prune (4 cases). 124 of the fungal extracts from apple were correctly identified (two with a score between 1.7 and 2.0 , the remaining 122 with a score $>2.0$ ). Of the fungal extracts from prune, 36 lead to the correct identification ( 4 with a score between 1.7 and 2.0, 32 with a score $>2.0$ ), and all the 56 fungal extracts from cherry were identified with a high score $(>2.0)$. These results confirmed that, using the extraction protocol presented here, the fruit host did not significantly alter MALDI-TOF MS spectra of the four Monilinia species. This approach thus established the reliable identification of M. fructicola, M. laxa and M. fructigena/M. polystroma by MALDI-TOF MS by using fungal material obtained directly from an infected host (Fig. 3).

Table 2 Summary of all MALDI-TOF MS results generated for this study

\begin{tabular}{|c|c|c|c|c|c|c|}
\hline & \multirow[t]{2}{*}{ Species } & \multirow[t]{2}{*}{ Extracts } & \multicolumn{2}{|c|}{ Correct ID (score) } & \multirow[t]{2}{*}{ Wrong ID } & \multirow[t]{2}{*}{ No ID } \\
\hline & & & $>2.0$ & $1.7-2.0$ & & \\
\hline \multirow[t]{3}{*}{ Agar } & M. fructicola & 30 & 30 & 0 & 0 & 0 \\
\hline & M. laxa & 22 & 19 & 1 & 1 & 1 \\
\hline & M. fructigena/M. polystroma & 36 & 30 & 3 & 0 & 3 \\
\hline \multirow[t]{3}{*}{ Apple } & M. fructicola & 25 & 23 & 0 & 0 & 2 \\
\hline & M. laxa & 25 & 24 & 0 & 0 & 1 \\
\hline & M. fructigena/M. polystroma & 80 & 75 & 2 & 0 & 3 \\
\hline \multirow[t]{3}{*}{ Prune } & M. fructicola & 7 & 5 & 1 & 0 & 1 \\
\hline & M. laxa & 7 & 3 & 1 & 1 & 2 \\
\hline & M. fructigena/M. polystroma & 33 & 24 & 2 & 3 & 4 \\
\hline \multirow[t]{3}{*}{ Cherry } & M. fructicola & 31 & 31 & 0 & 0 & 0 \\
\hline & M. laxa & 4 & 4 & 0 & 0 & 0 \\
\hline & M. fructigena/M. polystroma & 21 & 21 & 0 & 0 & 0 \\
\hline
\end{tabular}

Monilinia protein extracts were obtained from different sources (fungus grown on PDA plates or infected apples, prunes or cherries). Samples which did not yield a mass spectrum (less than $5 \%$ ) were excluded from this table. All species were identified with a microflex LT MALDI-TOF mass spectrometer (Bruker Daltonics GmbH) using the BioTyper software (Version 3.1, Bruker Daltonics $\mathrm{GmbH}$ ) and a custom-made main spectra (MSP) reference database 


\section{a}
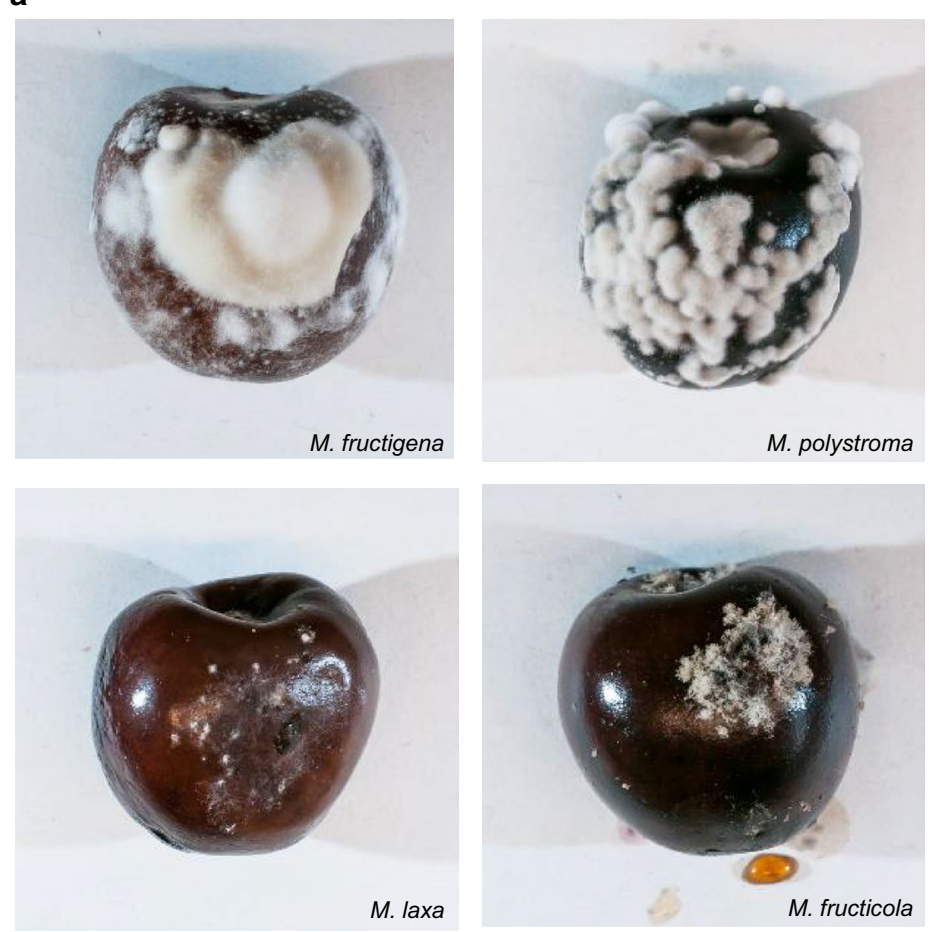

b

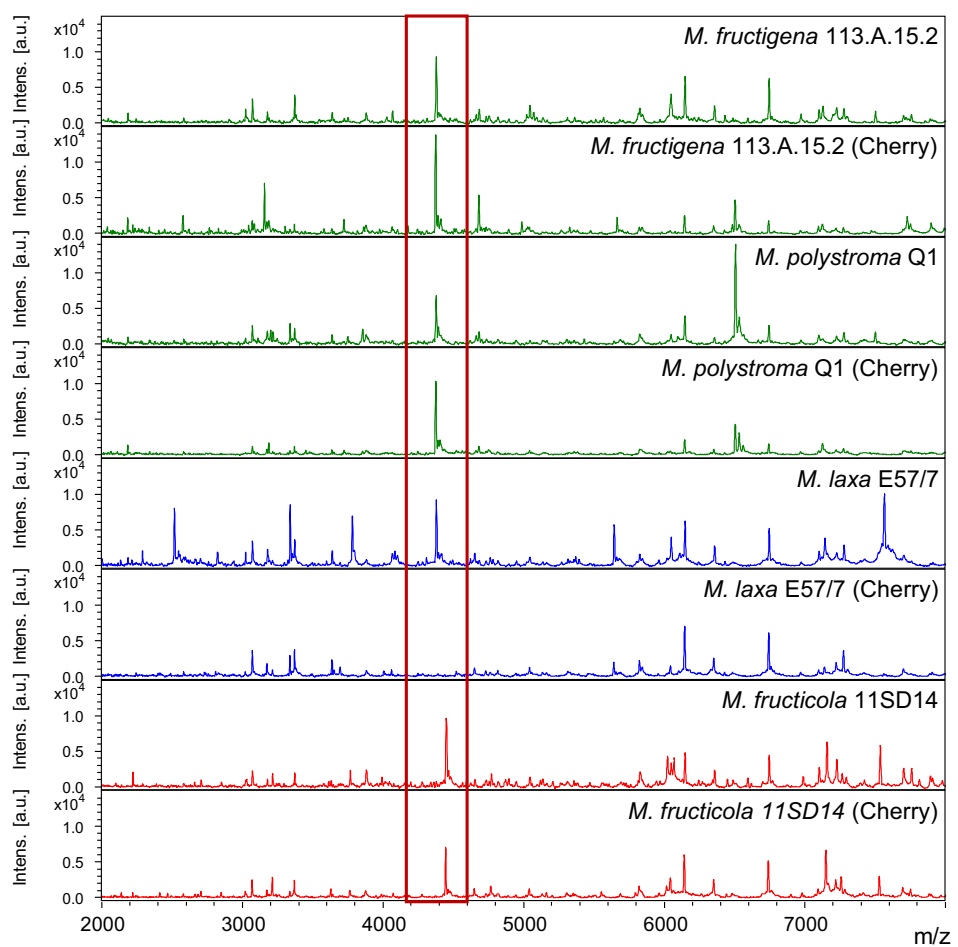

Fig. 2 Monilinia mycelium obtained from infected cherries can be directly used for MALDI biotyping. a Freshly harvested, ripe cherries were infected and colonized by all four species of Monilinia. b MALDI-TOF MS spectra for M. fructigena and M. polystroma (green), M. laxa (blue) and M. fructicola (red). For each species, a representative spectrum obtained from an in vitro culture and from infected cherries is shown 


\section{Characterization of a potential Monilinia low-mass peak biomarker by MALDI-TOF/TOF MS}

In the future, closely related species that are difficult to distinguish may be set apart employing species-specific biomarker peptides. The initial MALDI-TOF MS analyses of Monilinia species revealed prominent, isolated molecular average masses at approx. 4370 or $4450 \mathrm{Da}$, respectively, that seemed important for the separation of $M$. fructicola from the other species and offered the basis for a prompt identification of Monilinia species in general. To evaluate their potential as biomarkers, we have attempted to structurally characterize these mass peaks further by applying ziptip sample cleaning-up of fungal protein extracts (M. polystroma and $M$. fructicola) and subsequent MALDI-TOF/TOF MS analysis (Fig. 3). MALDI-TOF MS operating in the reflectron mode, instead of the linear mode, improved the resolution and revealed monoisotopic masses of 4373.3 and 4442.0 Da for M. polystroma and M. fructicola, respectively (Fig. 4a). Further structural characterization by the LIFT MALDI-TOF/TOF method resulted in characteristic fragmentation patterns (Fig. 4b), indicating that these peptides of the two Monilinia species differ spectrally only slightly from each other and may thus be closely related. These experiments documented that isolated low-mass peaks characteristic of Monilinia brown rot fungi can be directly subjected to MALDI-TOF/TOF MS analysis, without prior separation by chromatography, which may be used for developing more sophisticated biomarker analyses in the future.

\section{Discussion}

Microbial biotyping of intact cells by mass spectrometry has revolutionized the identification of microorganisms, in particular in the clinical setting, but also holds great promise as a new tool for basic research in microbiology and ecology [27]. Comparison of a newly acquired MALDI-TOF MS spectrum with reference spectra is able to identify an unknown organism with a very short time-to-result (minutes vs. hours or days for classical phenotypic or genotypic identification). The rapid processing and measurement, as well as the negligible requirement of consumables, make MALDI biotyping the method of choice for the routine identification of microorganisms, provided access to a mass spectrometer is guaranteed. In particular in clinical applications, MALDI biotyping is thus widely used and comprehensive databases of reference spectra exist for medically relevant microorganisms. However, while the method performs reliably for many bacteria, the MALDI-TOF MS-based identification of fungi is often more difficult and requires optimization of extraction and data acquisition protocols. Another major limitation for the rapid identification of bacteria and fungi is the cultivation step, which is usually required prior to MALDI-TOF MS analysis.

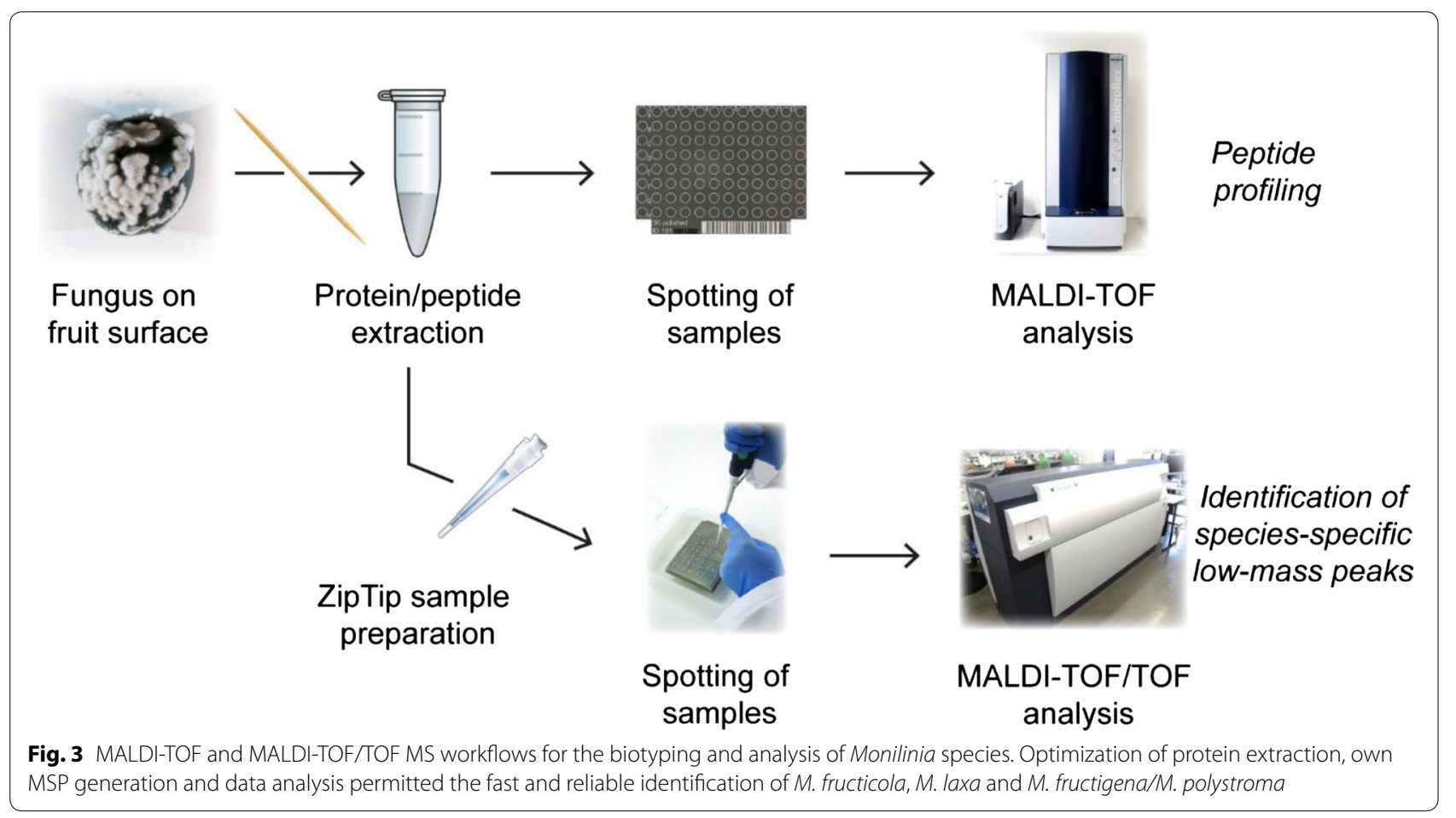


Until now, MALDI-TOF MS-based identification of fungi has been mainly used in medicine [28-34], but specific protocols to identify fungal plant pathogens have been developed and are being improved continuously [35]. For example, Monilinia brown rot fungi have been subjected to different electrophoresis methods as well as to MALDI-TOF MS analysis [22]. Similarly, downy and powdery mildew, caused by Bremia lactucae and Oidium neolycopersici, respectively, were identified by MALDITOF MS in vitro as well as on infected plant leaves [36]. Furthermore, MALDI-TOF MS, in combination with a DNA-based phylogenetic analysis, was used to compare and differentiate closely related, plant pathogenic Alternaria species [37] as well as a collection of 45 Brazilian isolates of the genus Clonostachys [38].

In the work presented here, MALDI biotyping alone was able to directly identify M. fructicola, M. laxa and $M$. fructigena/M. polystroma infections on apples, cherries and prunes, without a prior cultivation in vitro. The same approach also used to identify Monilinia brown rot from naturally infected apricots and prunes collected in Switzerland. As for the experimentally infected apples, cherries and prunes, Monilinia species were correctly identified (as confirmed by PCR) solely with material obtained from fruit (e.g. without prior cultivation) (data not shown). This indicates that the MALDI-based identification of natural Monilinia brown rot infections is possible as long as a particular lesion is caused by one fungal species. However, natural apricot and prune infections were almost exclusively due to M. laxa and M. polystroma, respectively, which prevented assessing the MALDI-based distinction between the four Monilinia brown rot species. Therefore, we only collected and show quantitative data obtained from experimentally infected fruits. The results obtained by these studies are in contrast to earlier reports, where a combination of MALDI-TOF MS and electrophoresis methods was suggested for the identification of Monilinia species on fruit samples, because the MALDI-TOF results were affected by the host [22]. It cannot be excluded that other hosts than apple, cherry or prune would affect the MALDI biotyping results and prevent a correct identification. However, the three hosts used in this study include different fruit types that minimally affected the performance of the MALDI biotyping. We thus expect that a correct MALDI biotyping with host-derived material is possible as long as the pathogen forms aerial mycelium and conidia on the host surface. We can only speculate on the factors that allowed the reliable identification of Monilinia brown rot fungi on host tissue by MALDI biotyping in this study. First of all, several isolates (18 altogether) were used to generate the MSP; the reference spectra for species identification. Importantly, the extraction procedure was identically performed for the processing of the samples used for the generation of MSP, for the fungal material cultivated in vitro, as well as for the fungal material from infected hosts. The extraction protocol itself has also been optimized by reducing the amount of fungal material that was extracted, the extraction of conidia and mycelium, and the addition of glass beads during the formic acid/acetonitrile extraction step. Finally, the parameters for the actual MALDITOF MS measurement, data processing and data analysis were adjusted (laser intensity, peak exclusion criterion, number of shots per spot, intensity correction for identification). In combination, this optimization led to a time-saving procedure and the reliable identification of M. fructicola, M. laxa and M. fructigena/M. polystroma. The fact that $M$. fructigena and $M$. polystroma could not be distinguished by the workflow described in this study underlines the close relatedness of these two species, which are set apart only by five base transitions in their ITS region [6]. It is thus laborious to distinguish these two species, even by molecular methods [7, 39]. The low intensity of most signals for $M$. polystroma, as well as the intense peak with a mass of $4377 \mathrm{Da}$ that was shared with $M$. fructigena (4378 Da), may explain the difficulty in separating these two species by MALDI biotyping (Fig. 1a).

By concentrating and purifying fungal extracts and subsequent MALDI-TOF/TOF MS analysis we have shown that prominent peptide peaks can be structurally characterized without preceding liquid chromatography. This workflow may generally serve as a suitable tool to identify species-specific low-mass peaks of fungal species and therefore contribute to the rapid and efficient identification of species that cannot be differentiated easily so far. The full identification of these peptides will not only allow identifying their biological function, but also to establish a rapid identification of fungi by screening for specific masses in selected reaction monitoring (SRM) experiments.

\section{Conclusion}

The work described here established a fast and reliable approach for the MALDI-TOF-based identification of four Monilinia brown rot fungi directly on infected fruits. This method not only represents an improvement of currently existing diagnostic protocols, but may also facilitate and enable basic research and field studies on the ecology of these important plant pathogens. 

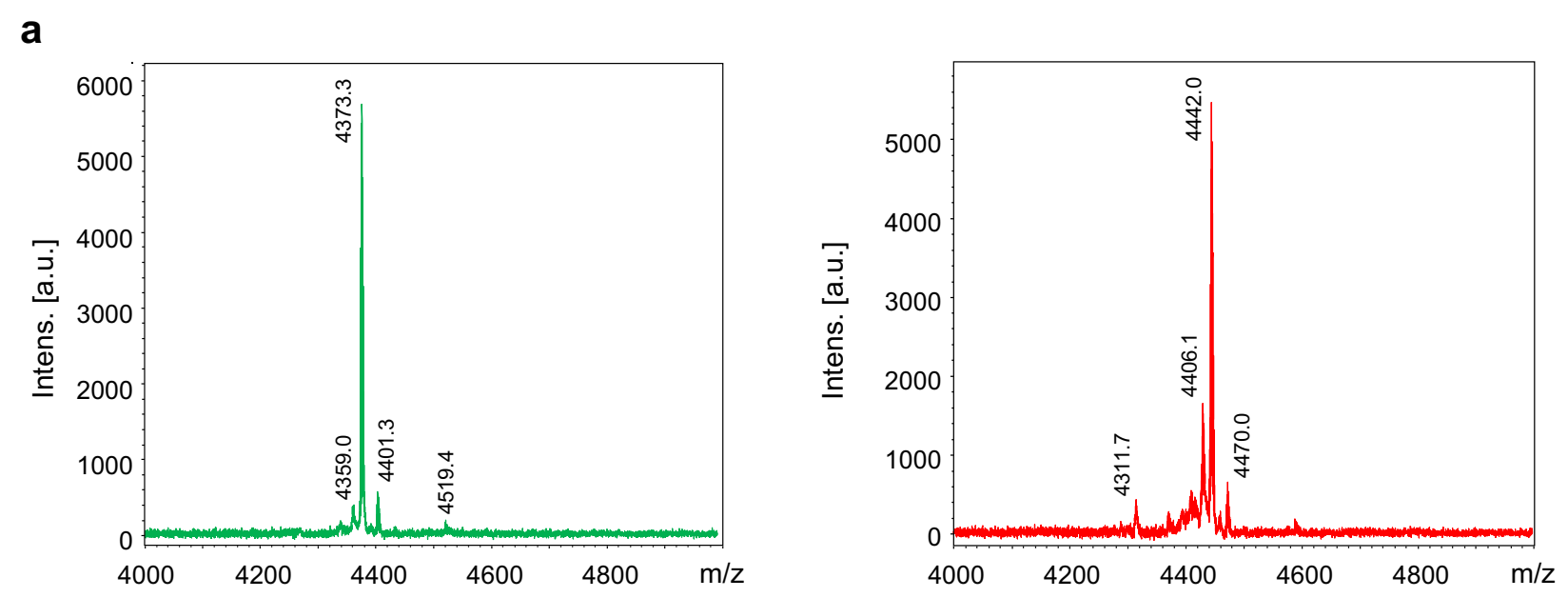

b
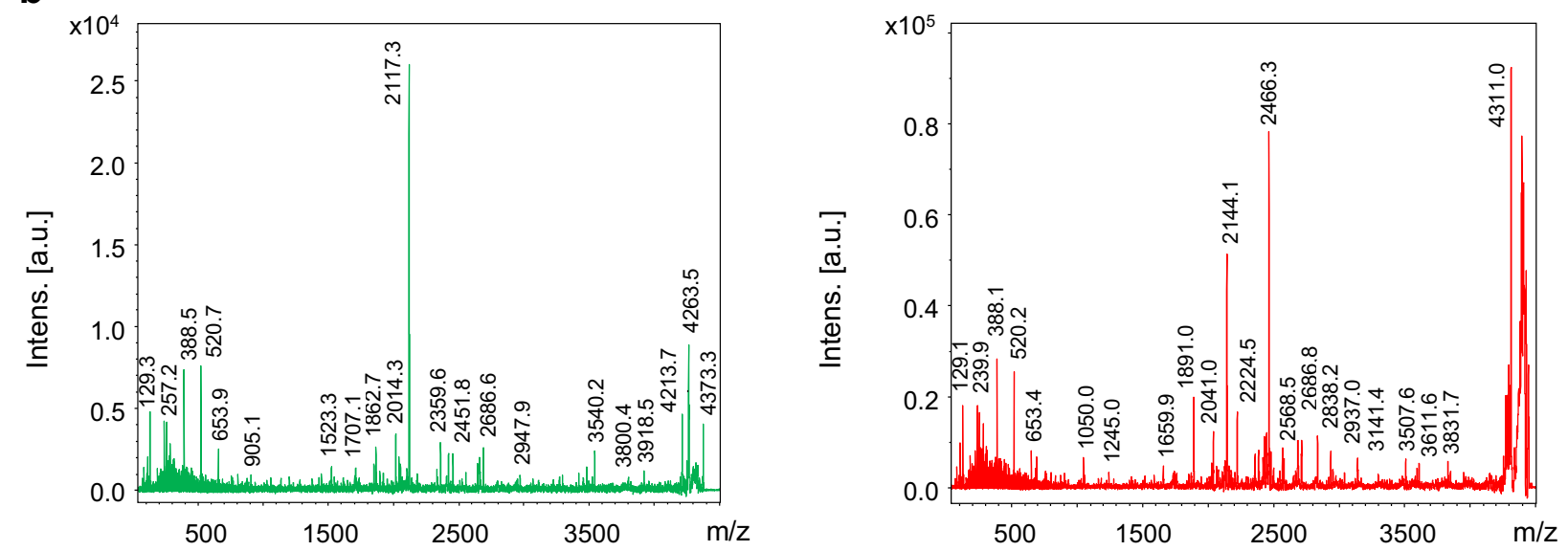

Fig. 4 MALDI-TOF/TOF MS of species-specific Monilinia peaks. a Species-specific monoisotopic masses for M. polystroma (green) and M. fructicola (red). b Fragment ions revealed for the masses of 4373.3 and 4442.0 Da, for M. polystroma (green) and M. fructicola (red), respectively, after LIFT fragmentation

\section{Authors' contributions}

FMF and MHB performed the lab work and all MALDI biotyping experiments. $\mathrm{DD}$ and RB performed MS/MS experiments, analysed the MS/MS data and wrote the corresponding sections of the manuscript. FMF and DD planned the study and FMF wrote the manuscript. All authors read and approved the final manuscript.

\section{Author details}

${ }^{1}$ Agroscope, Institute for Plant Production Sciences IPS, Schloss 1 , 8820 Wädenswil, Switzerland. ${ }^{2}$ Functional Genomics Center Zurich, ETH Zurich and University of Zurich, Winterthurerstr. 190, 8057 Zurich, Switzerland. ${ }^{3}$ Agroscope, Institute for Food Sciences IFS, Schloss 1, 8820 Wädenswil, Switzerland.

\section{Acknowledgements}

We thank Daniel Prata for his help with experimental work. Maria-Theresia Gekenidis and Stefanie Pfrunder are acknowledged for their support with MALDI-TOF MS measurements and data analysis. We recognize Eduard Holliger for his helpful and constructive feedback on the manuscript.

\section{Competing interests}

The authors declare that they have no competing interests.
Received: 18 November 2015 Accepted: 8 March 2016

Published online: 22 March 2016

\section{References}

1. Hrustic J, Mihajlovic M, Grahovac M, Delibasic G, Bulajic A, Krstic B, Tanovic B. Genus Monilinia on pome and stone fruit species. Pestic Phytomed (Belgrade). 2012;27(4):283-97.

2. Rungjindamai $N$, Jeffries $P, X u X-M$. Epidemiology and management of brown rot on stone fruit caused by Monilinia laxa. Eur J Plant Pathol. 2014;140(1):1-17.

3. Mari M, Martini C, Guidarelli M, Neri F. Postharvest biocontrol of Monilinia laxa, Monilinia fructicola and Monilinia fructigena on stone fruit by two Aureobasidium pullulans strains. Biol Control. 2012;60(2):132-40.

4. van Leeuwen GCM, Stein A, Holb IJ, Jeger MJ. Yield loss in apple caused by Monilinia fructigena (Aderh. \& Ruhl.) Honey, and spatio-temporal dynamics of disease development. Eur J Plant Pathol. 2000;106(6):519-28.

5. van Leeuwen GCM, Baayen RP, Holb IJ, Jeger MJ. Distinction of the Asiatic brown rot fungus Monilinia polystroma sp. nov. from M. fructigena. Mycol Res. 2002;106(04):444-51. 
6. Fulton CE, van Leeuwen GCM, Brown AE. Genetic variation among and within Monilinia specis causing brown rot of stone and pome fruits. Eur J Plant Pathol. 1999;105(5):495-500.

7. Hilber-Bodmer M, Knorst V, Smits THM, Patocchi A. First report of asian brown rot caused by Monilia polystroma on apricot in Switzerland. Plant Dis. 2012;96(1):146.

8. Hong C, Holtz BA, Morgan DP, Michailides TJ. Significance of thinned fruit as a source of the secondary inoculum of Monilinia fructicola in California nectarine orchards. Plant Dis. 1997;81(5):519-24.

9. De Cal A, Eguen B, Melgarejo P. Vegetative compatibility groups and sexual reproduction among Spanish Monilinia fructicola isolates obtained from peach and nectarine orchards, but not Monilinia laxa. Fungal Biol. 2014;118(5-6):484-94.

10. Chen Y, Zhou MG. Sexual recombination of carbendazim resistance in Fusarium graminearum under field conditions. Pest Manag Sci. 2009;65(4):398-403

11. Jänsch M, Frey JE, Hilber-Bodmer M, Broggini GAL, Weger J, Schnabel G, Patocchi A. SSR marker analysis of Monilinia fructicola from Swiss apricots suggests introduction of the pathogen from neighbouring countries and the United States. Plant Pathol. 2012;61(2):247-54.

12. Lane CR. A synoptic key for differentiation of Monilinia fructicola, M. fructigena and M. laxa, based on examination of cultural charcters. EPPO Bull. 2002;32(3):489-93.

13. OEPP/EPPO. Monilinia fructicola. EPPO Bull. 2009:39(3):337-43.

14. EFSA Panel on Plant Health (PLH). Pest risk assessment of Monilinia fructicola for the EU territory and identification and evaluation of risk management options. EFSA J. 2011;9(4):2119.

15. Hilber-Bodmer M, Bünter M, Patocchi A. First report of asian brown rot caused by Monilia fructicola on apricot in a Swiss orchard. Plant Dis. 2010;94(5):643.

16. EPPO A2. List of pests recommended for regulation as quarantine pests [http://www.eppo.int/QUARANTINE/listA2.htm].

17. Côté M-J, Tardif M-C, Meldrum AJ. Identification of Monilinia fructigena, M. fructicola, M. laxa, and Monilia polystroma on inoculated and naturally infected fruit using multiplex PCR. J Plant Dis. 2004;88(11):1219-25.

18. Anhalt JP, Fenselau C. Identification of bacteria using mass spectrometry. Analytical Chem. 1975;47(2):219-25.

19. Fenselau C, Demirev PA. Characterization of intact microorganisms by MALDI mass spectrometry. Mass Spectrom Rev. 2001;20(4):157-71.

20. Sauer S, Kliem M. Mass spectrometry tools for the classification and identification of bacteria. Nat Rev Microbiol. 2010;8(1):74-82.

21. Evason DJ, Claydon MA, Gordon DB. Exploring the limits of bacterial identification by intact cell-mass spectrometry. J Am Soc Mass Spectrom. 2001;12(1):49-54

22. Horka M, Kubesova A, Salplachta J, Zapletalova E, Horky J, Slais K. Capillary and gel electromigration techniques and MALDI-TOF MS—suitable tools for identification of filamentous fungi. Anal Chim Acta. 2012;716:155-62.

23. Kawasaki ES. Sample preparation from blood, cells, and other fluids. In: Innis MA, Gelfand DH, Sninsky JJ, White TJ, editors. PCR Protocols: a guide to methods and applications. San Diego: Academic Press; 1990. p. 146-52.

24. Gardes M, Bruns TD. ITS primers with enhanced specificity for basidiomycetes-application to the identification of mycorrhizae and rusts. Mol Ecol. 1993;2(2):113-8.
25. White TJ, Bruns TD, Lees S, Taylor JW. Amplification and direct sequencing of fungal ribosomal RNA genes for phylogenetics. In: Innis MA, Gelfand DH, Sninsky JJ, White TJ, editors. PCR Protocols: a guide to methods and applications. San Diego: Academic Press; 1990. p. 315-22.

26. Neubert H, Halket JM. Fernandez Ocana M, Patel RK: MALDI post-source decay and LIFT-TOF/TOF investigation of alpha-cyano-4-hydroxycinnamic acid cluster interferences. J Am Soc Mass Spectrom. 2004;15(3):336-43.

27. Ferreira L, Sanchez-Juanes F, Garcia-Fraile P, Rivas R, Mateos PF, MartinezMolina E, Gonzalez-Buitrago JM, Velazquez E. MALDI-TOF mass spectrometry is a fast and reliable platform for identification and ecological studies of species from family Rhizobiaceae. PLoS One. 2011;6(5):e20223.

28. De Carolis E, Posteraro B, Lass-Florl C, Vella A, Florio AR, Torelli R, Girmenia C, Colozza C, Tortorano AM, Sanguinetti M, et al. Species identification of Aspergillus, Fusarium and Mucorales with direct surface analysis by matrixassisted laser desorption ionization time-of-flight mass spectrometry. Clin Microbiol Infect. 2012;18(5):475-84.

29. Nenoff P, Erhard M, Simon JC, Muylowa GK, Herrmann J, Rataj W, Graser Y. MALDI-TOF mass spectrometry - a rapid method for the identification of dermatophyte species. Med Mycol. 2013;51(1):17-24.

30. Ling H, Yuan Z, Shen J, Wang Z, Xu Y. Accuracy of matrix-assisted laser desorption ionization-time of flight mass spectrometry for identification of clinical pathogenic fungi: a meta-analysis. J Clin Microbiol. 2014;52(7):2573-82.

31. De Carolis E, Vella A, Vaccaro L, Torelli R, Posteraro P, Ricciardi W, Sanguinetti $M$, Posteraro B. Development and validation of an in-house database for matrix-assisted laser desorption ionization-time of flight mass spectrometry-based yeast identification using a fast protein extraction procedure. J Clin Microbiol. 2014;52(5):1453-8.

32. Posteraro B, De Carolis E, Vella A, Sanguinetti M. MALDI-TOF mass spectrometry in the clinical mycology laboratory: identification of fungi and beyond. Expert Rev Proteomics. 2013;10(2):151-64.

33. Bader O. MALDI-TOF-MS-based species identification and typing approaches in medical mycology. Proteomics. 2013;13(5):788-99.

34. Del Chierico F, Masotti A, Onori M, Fiscarelli E, Mancinelli L, Ricciotti G, Alghisi F, Dimiziani L, Manetti C, Urbani A, et al. MALDI-TOF MS proteomic phenotyping of filamentous and other fungi from clinical origin. J Proteomics. 2012;75(11):3314-30.

35. Chalupova J, Raus M, Sedlarova M, Sebela M. Identification of fungal microorganisms by MALDI-TOF mass spectrometry. Biotechnol Adv. 2014;32(1):230-41.

36. Chalupova J, Sedlarova M, Helmel M, Rehulka P, Marchetti-Deschmann M, Allmaier G, Sebela M. MALDI-based intact spore mass spectrometry of downy and powdery mildews. J Mass Spectrom. 2012;47(8):978-86.

37. Brun S, Madrid H, Van Gerrits Den Ende B, Andersen B, Marinach-Patrice C, De Hoog GS. Multilocus phylogeny and MALDI-TOF analysis of the plant pathogenic species Alternaria dauci and relatives. Fungal Biol. 2013;117(1):32-40.

38. Abreu LM, Moreira GM, Ferreira D, Rodrigues-Filho E, Pfenning LH. Diversity of Clonostachys species assessed by molecular phylogenetics and MALDI-TOF mass spectrometry. Fungal Biol. 2014;118(12):1004-12.

39. Chalkley D: Invasive fungi. Asiatic brown fruit rot - Monilinia polystroma. In.: Systematic Mycology and Microbiology Laboratory, ARS, USDA. 